\section{BMJ Open \\ Respiratory \\ Research}

\title{
Reliable EGFR mutation testing in ultrasound-guided supraclavicular lymph node fine-needle aspirates: a cohort study with diagnostic performance analysis
}

\author{
Amir Awwad, ${ }^{1}$ Sandeep Tiwari, ${ }^{1}$ Vishakha Sovani, ${ }^{2}$ David R Baldwin, ${ }^{3}$ \\ Maruti Kumaran $^{1}$
}

To cite: Awwad A, Tiwari S, Sovani V, et al. Reliable EGFR mutation testing in ultrasound-guided supraclavicular lymph node fine-needle aspirates: a cohort study with diagnostic performance analysis. BMJ Open Resp Res 2015;2:e000075

doi:10.1136/bmjresp-2014000075

A preliminary data analysis (earlier version of the abstract) has previously been accepted and presented (poster) at the 11th Annual British Thoracic Oncology Group Conference, 2013: Diagnosis and Staging Lung Cancer 2013.

Received 5 December 2014 Revised 17 April 2015 Accepted 20 April 2015

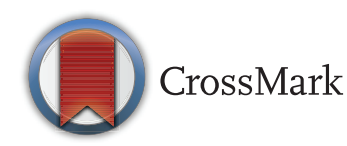

For numbered affiliations see end of article.

Correspondence to Dr Amir Awwad; amir.awwad@nhs.net

\section{ABSTRACT}

Introduction: $15-30 \%$ of patients with lung cancer will have supraclavicular and cervical lymphadenopathy (SCLN). Ultrasound (US)-guided fine-needle aspiration (FNA) cytology is regarded as an effective diagnostic tool in small size lymph nodes (LNs) and impalpable positron emission tomography detected nodes. We evaluated our diagnostic service performance in relation to the adequacy of samples for epidermal growth factor receptor (EGFR) mutation.

Methods: Retrospective data analysis from electronic records, searching for all suspected lung cancer referrals that underwent US of the neck \pm FNA, over a continuous period of 4 years.

Results: Of 306 cases with suspected lung cancer referred to our department for US FNA of SCLN, 228 patients underwent the procedure. Of the remaining 78 patients, LNs were not detected in 52 cases and appeared benign in 26. Cytological diagnosis was established in 171 patients $(75 \%)$ for treatment decisions without further investigations. The remaining 57 patients had further investigations; 45 reconfirmed the US-guided FNA diagnosis. The average LN size was $12.9 \mathrm{~mm}$, and positive cytology was obtained in LNs ranging from 3 to $45 \mathrm{~mm}$. Of 57 adenocarcinoma cases, 34 were tested for EGFR confirming 4 positive, 25 negative and 5 insufficient samples.

No complications were recorded.

Conclusions: US-guided FNA of SCLN remains an important diagnostic tool in lung cancer. Adequate tissue can be obtained for reliable diagnosis from LNs and for EGFR mutational analysis, without the need for more invasive and expensive investigations in more than $80 \%$ of cases.

\section{INTRODUCTION}

Fifteen to 30 per cent of patients with lung cancer will have supraclavicular lymphadenopathy (SCLN) and anterior cervical lymph nodes (LNs). ${ }^{12}$ Ultrasound (US)-guided fine-

\section{KEY MESSAGES}

In lung cancer, US guided FNA of SCLN is safe and easy standard test for definitive histopathological confirmation.

- It is also a reliable test to obtain an adequate sample for accurate diagnostic and EGFR mutational analysis.

- This 4-years diagnostic cohort study provides new evidence on the relevant potential of US guided FNA of SCLN in lung cancer molecular testing.

needle aspiration (FNA) cytology (FNAC) is known to be an effective diagnostic tool in small size supraclavicular or neck LNs as well as in impalpable positron emission tomography (PET)-detected nodes. ${ }^{3}{ }^{4}$ The latest algorithm of the National Institute for Health and Care Excellence (NICE) guidance published in April 2011 incorporates the use of US of the neck to obtain cytology (figure 1B). It clearly indicates that clinicians should offer US of the neck with an intention to sample any visibly abnormal LNs or non-US-guided transbronchial needle aspiration (TBNA) to patients with a high probability of mediastinal malignancy (LNs $>20 \mathrm{~mm}$ maximum short axis on CT. ${ }^{5}$ It also states that US of the neck should be offered with biopsy of visible LNs to patients who have neck nodes detected by initial CT. If CT is negative, non-US-guided TBNA or endobronchial US (EBUS)-guided TBNA or endoscopic US-guided FNA is recommended. ${ }^{5}$

Testing for epidermal growth factor receptor (EGFR) mutation, and other mutations, has now become an important step in the treatment decision pathway in patients with non-small cell lung cancer (NSCLC). ${ }^{6}$ This is 
because there are now three EGFR tyrosine kinase inhibitors that are currently approved by NICE following Single Technology Appraisals. ${ }^{7-10}$ Cytological samples have been shown to be adequate for EGFR mutational testing, although initially there was concern that the samples would be too small. ${ }^{7}$ However, to the best of our knowledge, there has been no confirmation that the US-guided FNA of the neck and SCN samples are adequate for EGFR mutation detection. Therefore, the objectives of this study are (1) to investigate the utility of US-guided SCL FNAC to provide adequate samples for EGFR testing from impalpable neck nodes and (2) to assess the diagnostic performance of SCL FNAC in suspected lung cancer compared with other gold standard and/or invasive investigations.

\section{MATERIALS AND METHODS}

\section{Review of records}

A retrospective analysis of a prospectively collated database was carried out on all patients referred to Nottingham University Hospitals, a tertiary referral centre in the UK, for US-guided SCLN FNA using the hospital electronic systems and covering the period between 31 October 2009 and 1 November 2013. Our electronic research records included a Computerised Radiology Information System (CRIS) used to report imaging and interventional procedures in conjunction with the Picture Archiving and Communication System (PACS), and cross-matched with patients' clinical notes and histological reports found on the Nottingham Information System (NotIS). Records were included in the analysis where the procedure was performed for patients with lung cancer who either had image-detected nodes (usually CT), or where there was bulky mediastinal lymphadenopathy. We identified a cohort of patients $(n=306)$ with suspected lung cancer referred to our radiology department for a US of the neck examination with a view to obtaining an FNAC. Data recorded included the date of procedure, US findings, size and morphology of the LNs, and the need for further investigation. Pre-FNA CT reports were collated and analysed. Diagnostic accuracy results were expressed in sensitivity, specificity and likelihood ratios in compliance with the published guidelines and research checklist stated in the STARD initiative endorsed by the EQUATOR network. ${ }^{11}$ For the purpose

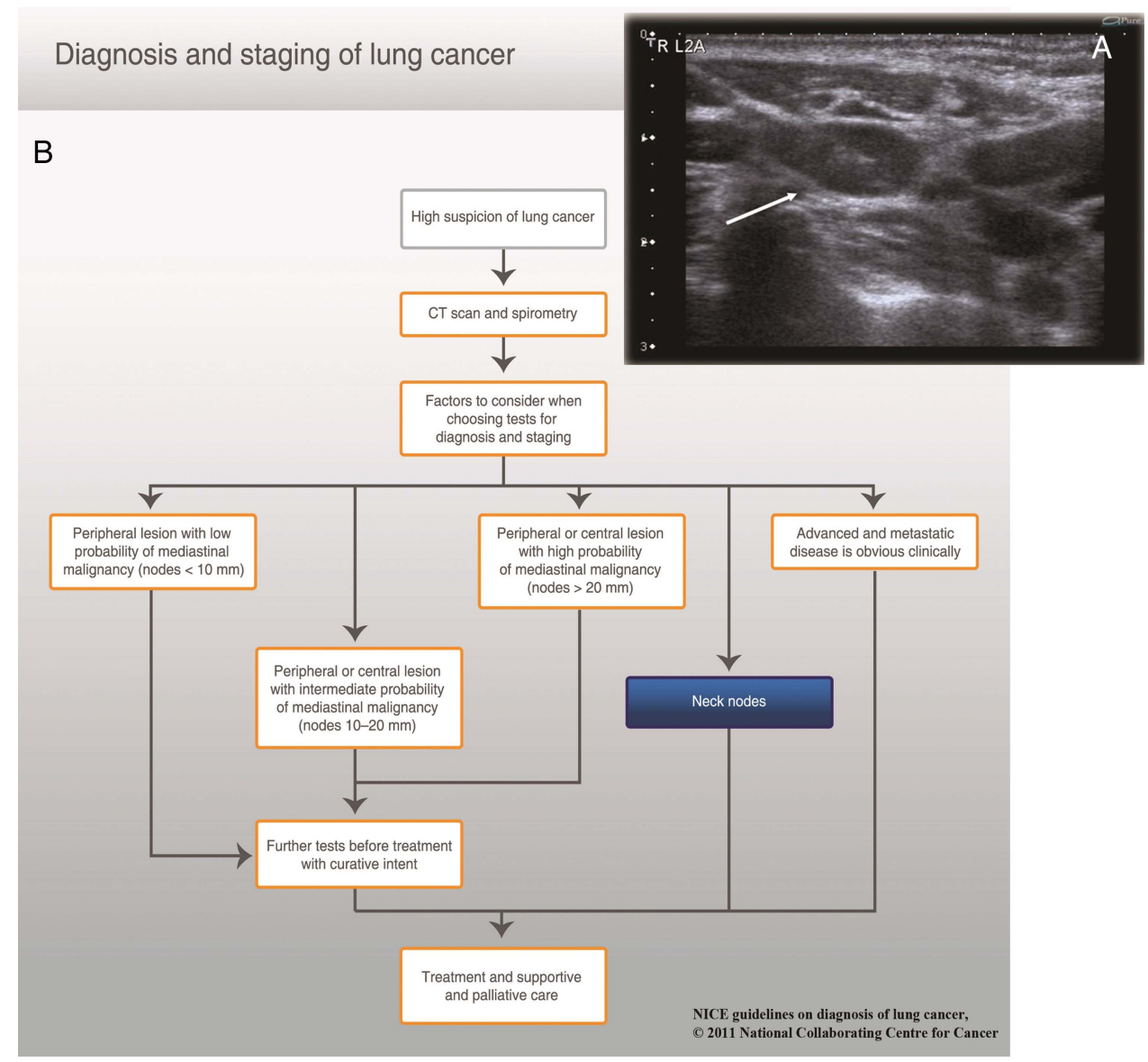

Figure 1 (A) Cross-sectional (axial) sonographic image showing a 21-gauge fine-needle aspiration cytology needle introduced in a $12 \mathrm{~mm}$ supraclavicular lymph node (white arrow). (B) Adapted version of the updated 2011 diagnostic pathway published by the National Collaboration Centre for Cancer (NICE Clinical Guidelines, CG121 April 2011) emphasising on the sole role of neck nodes in reaching a diagnosis decision leading to treatment (curative or palliation). All rights reserved. ${ }^{5}$ 
of reporting this study, US-guided SCLN FNA is considered the 'Index Test', and other methods used for obtaining core tissue biopsy are regarded in combination as the 'Reference Tests'.

\section{FNAC procedure}

All examinations and procedures were performed and/ or supervised by a consultant chest radiologist. The procedure was first explained to the patient, then informed consent was obtained, and finally the WHO checklist was completed. Preferably, patients were lying supine with a small pillow under the shoulders to allow a degree of neck hyperextension; otherwise, in a few unfit patients, the erect position was adopted. The US examination of the neck was used to identify the most amenable SCL and/or neck LNs for sampling based on morphological assessment by the operating radiologist.

During the initial US assessment preceding potential FNAC sampling, a set of sonographic features was used to suggest a benign and abnormal appearance of SCL LNs. Those were predefined by the chest radiologist supervising and/or performing the procedure. For example, benign features of an SCL LN are demonstrated (1) if the nodes have preserved fatty hilum, (2) lentiform morphology, (3) defined margins and (4) no hazy or ill-defined surrounding fatty planes. Thus, nodal size enlargement is not always a rule. Also, a noticeable distortion of the normal hilar flow and/or the presence of a predominant compensatory capsular flow were suspicious features of disease involvement. In multiple/ bilateral detection of SCL LNs, selection for FNAC can, to some degree, be governed by the safest approach and easiest direct access window without serious risks to the supraclavicular neurovascular structures.

Sterile technique was observed during the procedure and $2-5$ millilitres of $1 \%$ lidocaine was used to anaesthetise the skin. A 21-gauge needle with capillary technique was used to obtain the FNA sample with at least two passes under US guidance (figure 1A). The aspirated material contained within the needle or its hub would be immediately flushed and stored in a CytoRich Red (Becton Dickinson, Franklin Lakes, New Jersey, USA) bottle and sent to the histopathology department. Abnormal SCL LNs were defined by their atypical sonographic and morphological appearance as well as their size. Short-axis measurements of aspirated nodes and hilar blood Doppler flow were also reported.

\section{EGFR mutation analysis}

In current practice, the most reported techniques to test for EGFR mutations are reliant on PCR. ${ }^{712}$ Three highly sensitive methods are referred to in literature which would either employ the Peptide Nucleic Acid-Locked Nucleic Acid PCR clamp, the Cycleave method and/or the PCR invader. ${ }^{9} 13$ All are capable of yielding DNA positional defects with a $1 \%$ ratio of cancer cells in a specimen. ${ }^{14}$ However, quantification and qualitative analysis of extracted DNA samples in our cohort is beyond the remit of our report. In our institution laboratories, we use pyrosequencing, which is an assay based on nested PCR for the characterisation of these mutations on formalin-fixed and paraffin-embedded tumour tissue (added advantage) as well as cytospins from FNAs. The yield of malignant cells on aspirated material is often variable. All samples

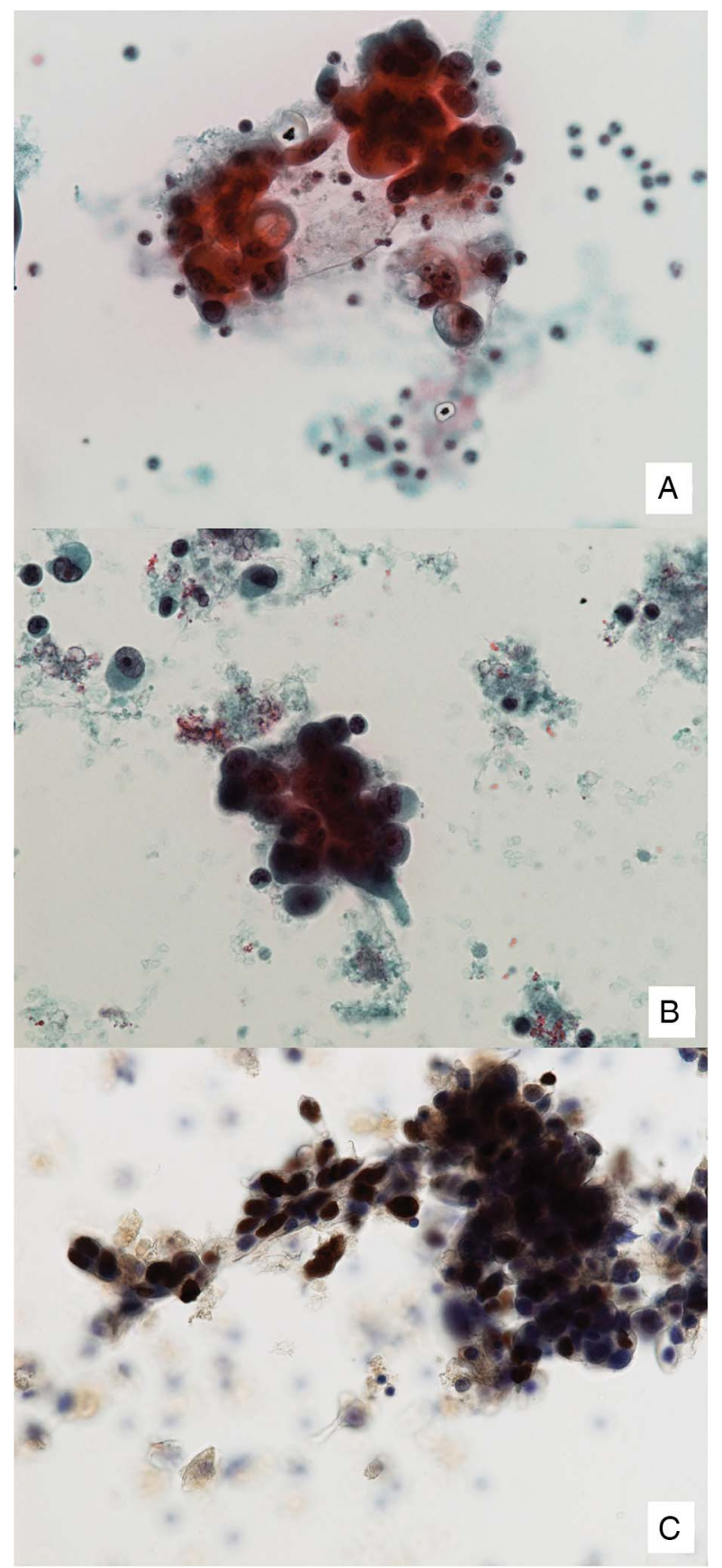

Figure 2 (A-C) Electron microscopic images obtained from two different cases, (A) a cluster of large malignant cells with cytoplasmic mucin vacuoles consistent with metastatic adenocarcinoma (Pap stain, $\times 40$ magnifications). (B) Large malignant cells with vesicular nuclei and prominent nucleoli consistent with metastatic non-small cell carcinoma (Pap stain, $\times 40$ magnifications). (C) Thyroid transcription factor 1 (TTF1, ×20 magnification) immunostaining slide showing nuclear positivity confirming metastatic adenocarcinoma. 
were first analysed by specialist pulmonary pathologists to classify tumours according to the WHO classification. Where the diagnosis of adenocarcinoma was not possible on the initial stains, samples were spun down to make into a cell block. The latter was found more suitable for immunohistochemical analysis and EGFR testing, especially as it is more predictive of the percentage of malignant cells in paucicellular samples. DNA was extracted using the manufacturer's guidelines and subjected to nested PCR to achieve the required amplification (see figure 2A-C for more details).

\section{RESULTS}

Three hundred and six patients (149 women, 157 men) were referred to the radiology department (median age of 68 years (range 35-95)) for US of the neck with a view to perform an FNA procedure if feasible during their US assessment. Out of those 306 patients, FNA was not performed in $26 \%(n=78)$ of patients either because US did not detect LNs $(n=52)$ or due to a benign morphological appearance $(n=26)$. In the remainder of patients $(n=228)$ who underwent the procedure, the average size of sampled LNs was $12.9 \mathrm{~mm}$ in the short
Figure 3 (A) A pie chart demonstrating the size ranges of aspirated SCLN (total $\mathrm{n}=228$ ). The large percentage $(\sim 41 \%)$ of sampled LNs is smaller than $10 \mathrm{~mm}$ in the short axis $(n=92)$.

(B) A bar chart demonstrating the proportions of positive (specifically for lung cancer) and negative SCLN according to the aspirated LNs sizes. (C) A pie chart illustrating the distribution of diagnoses made using ultrasound-guided FNA of SCLN (WHO 2004). FNA, fine-needle aspiration; LN, lymph node; NSCLC, non-small cell lung cancer; TB, tuberculosis; RCC, renal cell carcinoma; SCLN, supraclavicular and cervical lymphadenopathy.
A

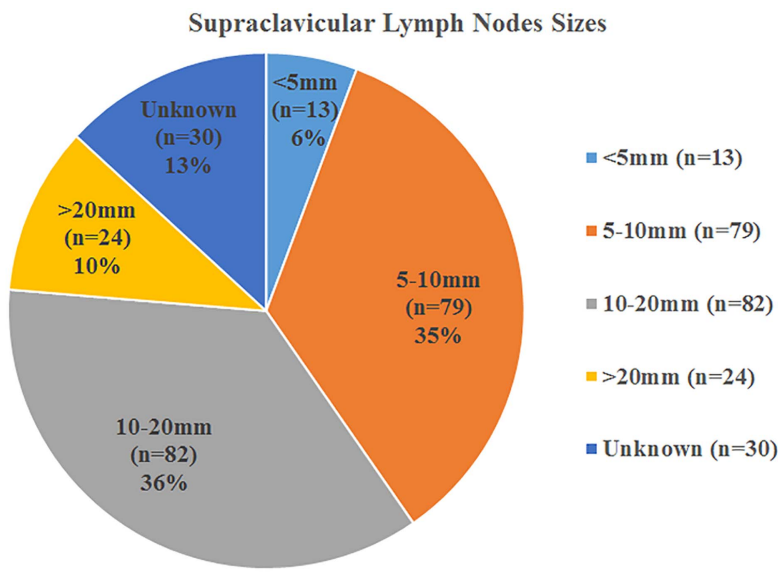

B
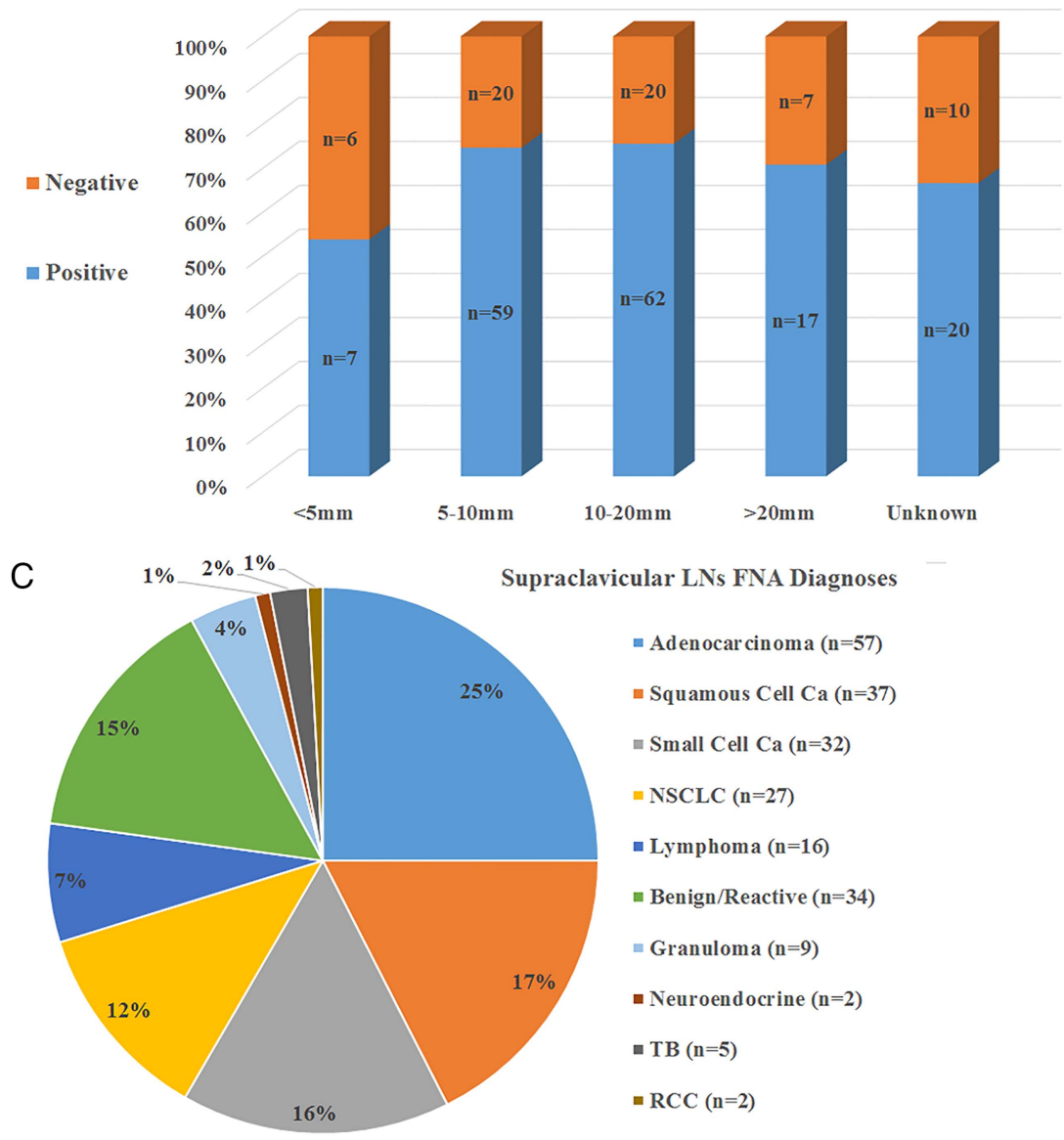
Table 1 List of further diagnostic investigations of lung cancer $(n=57)$ undertaken in the cohort

\begin{tabular}{ll}
\hline $\begin{array}{l}\text { Further investigations } \\
\text { (Reference Tests) }\end{array}$ & $\begin{array}{l}\text { Number of } \\
\text { patients }(\mathbf{n}=\mathbf{5 7})\end{array}$ \\
\hline Bronchial washings & 16 \\
SCL/mediastinal LN biopsy (non-US & 23 \\
guided) & \\
Lung biopsy & 8 \\
Pleural fluid cytology & 4 \\
Others (tongue, splenic, liver, rib biopsy) & 6 \\
\hline LN, lymph node; US, ultrasound. &
\end{tabular}

axis (range $3-45 \mathrm{~mm}$ ). Ninety-two $(41 \%)$ nodes measured less than $10 \mathrm{~mm}$ (subcentimetre) in their short axis. More than two-thirds ( $72 \%$ ) of those subcentimetre nodes were positive (figure $3 \mathrm{~A}-\mathrm{B}$ ).

Cytological diagnosis was established in $75 \%$ of patients $(n=171)$ based on SCL LNs FNA results without any further investigations required for treatment decisions. The remaining $25 \% \quad(n=57)$ of cases also had further investigations (Reference Tests, described in table 1$)$; approximately $70 \%(\mathrm{n}=40)$ of these cases were true positives and $9 \% \quad(\mathrm{n}=5)$ were true negatives (table 2A). Diagnostic accuracy analysis showed a $77 \%$ sensitivity and $100 \%$ specificity of FNA results (all further tests were used as the reference standard), with a positive predictive value of $100 \%$ and negative predictive value of $30 \%$. All index and reference tests results were accessible (unmasked) to either radiologists or pathologists; for more details on the individual reference tests (see tables 1-3). A final flow chart showing the distribution of histopathological results from US-guided SCLN FNA (the index test) and other reference standard tests is included (figure 4).

The highest proportion of the histological types was adenocarcinoma $(25 \%)$, followed by squamous cell carcinoma (16\%) and small cell carcinoma (14\%) (figure 3C). Of 57 adenocarcinomas, 34 were tested for EGFR mutational analysis with $5(\sim 15 \%)$ samples found insufficient for analysis. Subsequently, molecular analysis was confirmed on $29(\sim 85 \%)$ patients, 4 of whom tested positive for EGFR mutation.

In those unsampled cases where FNAC was not performed due to the above, we have further also checked their follow-up to show the following (hard data evidence):

\section{When 'No Cervical LN' was seen $(n=52)$}

Further investigations showed:

$\mathrm{n}=20$, confirming lung cancer;

$\mathrm{n}=2$, confirming non lung cancer $(\mathrm{TB}+$ sarcoidosis $)$;

$\mathrm{n}=4$, normal;

$\mathrm{n}=26$, no further tests.

\section{When 'Benign-Appearing Nodes' were found $(n=26)$}

Further investigations showed:

$\mathrm{n}=4$, confirming lung cancer;

$\mathrm{n}=1$, confirming non-lung cancer (non-Hodgkin's lymphoma);

$\mathrm{n}=4$, benign/reactive;

$\mathrm{n}=17$, no further tests.

Those patients $(n=20)$ with a subsequent diagnosis of lung cancer did not have nodes at the time of US-guided FNAC, and four had nodes that appeared benign. All non-sampled cases have been followed up for a minimum of 18 months, and none have developed malignancy.

\section{DISCUSSION}

This retrospective study has reviewed the everyday performance of US FNA of SCLN samples in the diagnosis of lung cancer, as recommended by the 2011 update of the NICE guideline on the management of lung cancer. This study showed that these samples were adequate for

Table 2 The $2 \times 2$ table for US-guided FNA SCLN results compared with lung cancer diagnosis made by all further investigations (Reference Tests), and with diagnosis made by the top 3 standard diagnostic reference tests (core LN biopsy, bronchial washings and lung biopsy)

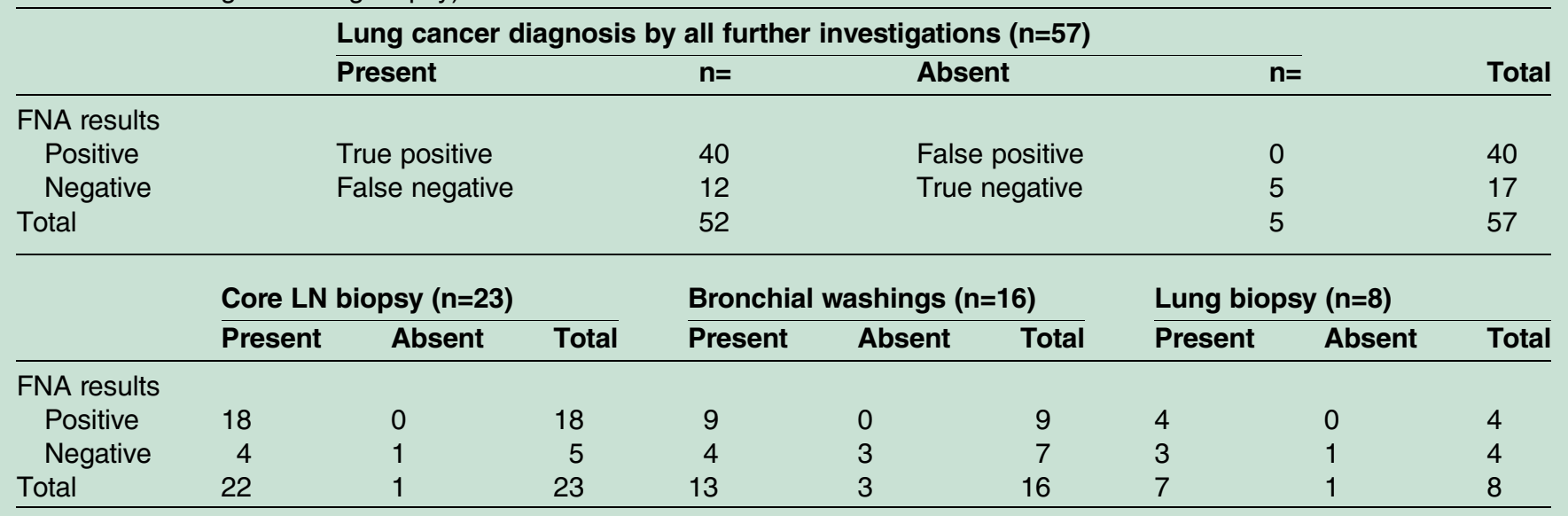

FNA, fine-needle aspiration; LN, lymph node; SCLN, supraclavicular and cervical lymphadenopathy; US, ultrasound. 
Table 3 Diagnostic performance with $95 \% \mathrm{Cl}$ of US-guided FNA SCLN results compared with lung cancer diagnosis made by all other further investigations in the first row (Reference Tests) and compared with the top 3 standard diagnostic reference tests (core LN biopsy, bronchial washings and lung biopsy)

\begin{tabular}{|c|c|c|c|c|c|c|}
\hline US-guided FNA vs & $\begin{array}{l}\text { Sensitivity \% } \\
(95 \% \mathrm{Cl})\end{array}$ & $\begin{array}{l}\text { Specificity \% } \\
(95 \% \text { Cl) }\end{array}$ & PPV \% (95\% Cl) & NPV \% (95\% Cl) & PLR & NLR \\
\hline All further tests & 76.9 (63.2 to 87.5$)$ & 100 (47.9 to 100$)$ & $100(91.1$ to 100$)$ & $29.4(10.4$ to 55.9$)$ & NA & $0.23(0.2$ to 0.4$)$ \\
\hline Core LN biopsy & 81.2 (59.7 to 94.7$)$ & 100 (16.6 to 100$)$ & 100 (81.3 to 100$)$ & 20 (3.3 to 71.2$)$ & NA & $0.18(0.1$ to 0.5$)$ \\
\hline Bronchial washings & 69.2 (38.6 to 90.7$)$ & 100 (30.5 to 100$)$ & 100 (66.2 to 100$)$ & 42.9 (10.4 to 81.3$)$ & NA & $0.3(0.1$ to 0.7$)$ \\
\hline Lung biopsy & 57.1 (18.8 to 89.6$)$ & 100 (16.6 to 100$)$ & $100(40.2$ to 100$)$ & 25 (4.1 to 79.7$)$ & NA & $0.4(0.2$ to 1.0$)$ \\
\hline
\end{tabular}

the purpose of EGFR mutation testing in $85 \%$ of samples tested.

The presence of a chest radiologist at the multidisciplinary team (MDT) meetings would trigger the referral of cases with impalpable SCL LNs for FNAC sampling. According to the National Institute for Health and Care Excellence (NICE) lung cancer guidelines, ${ }^{5}$ the MDT decision is essentially based on imaging findings (CT scan) showing a burden of mediastinal adenopathy (abnormal morphology with short-axis enlargement $>1 \mathrm{~cm}$ ) with presence or suspicion of SCL LNs on CT scan. The above ensures that all patients with bulky mediastinal nodes will then undergo US of the neck (preferred strategy number 13). During the whole study period, the sonographic findings of normal neck nodes have always proceeded after imaging detection of bulky mediastinal adenopathy and not the other way round.

US of the neck \pm FNA is reported to have $99 \%$ specificity and 33-50\% sensitivity in populations with intermediate to high prevalence of cancer, while there are no available reports on the accuracy in populations with a lower prevalence. Our experience and study findings are compatible with these reports as the prevalence of malignancy in our population was high. ${ }^{15}$

Current NICE guidelines recommend (figure 1B) referral for US of the neck \pm biopsy where neck lympadenopathy is detected on CT or clinically, and where there are multiple bulky mediastinal nodes. ${ }^{5}$ If the diagnostic accuracy that we have achieved can be replicated in other clinical services, this suggests that US neck might be routinely the first investigation rather than a choice of TBNA, which is more invasive. It is noted that the existing pathway recommended this choice on the basis of only a limited case series and expert opinion. ${ }^{5}$ While this could also be classed as a limited case series, the findings of the present study add to the evidence for US of the neck as a preferred test, but ideally a multicentre study is needed to resolve the uncertainty. On a similar level of evidence (case series), the latest guidelines on lung cancer management from the Scottish Intercollegiate Guidance Network (SIGN 137) incorporates the test in the staging investigations of lung cancer. $^{4{ }^{15-17}}$ Specifically, it recommends US of the neck FNA for the pathological diagnosis and staging of

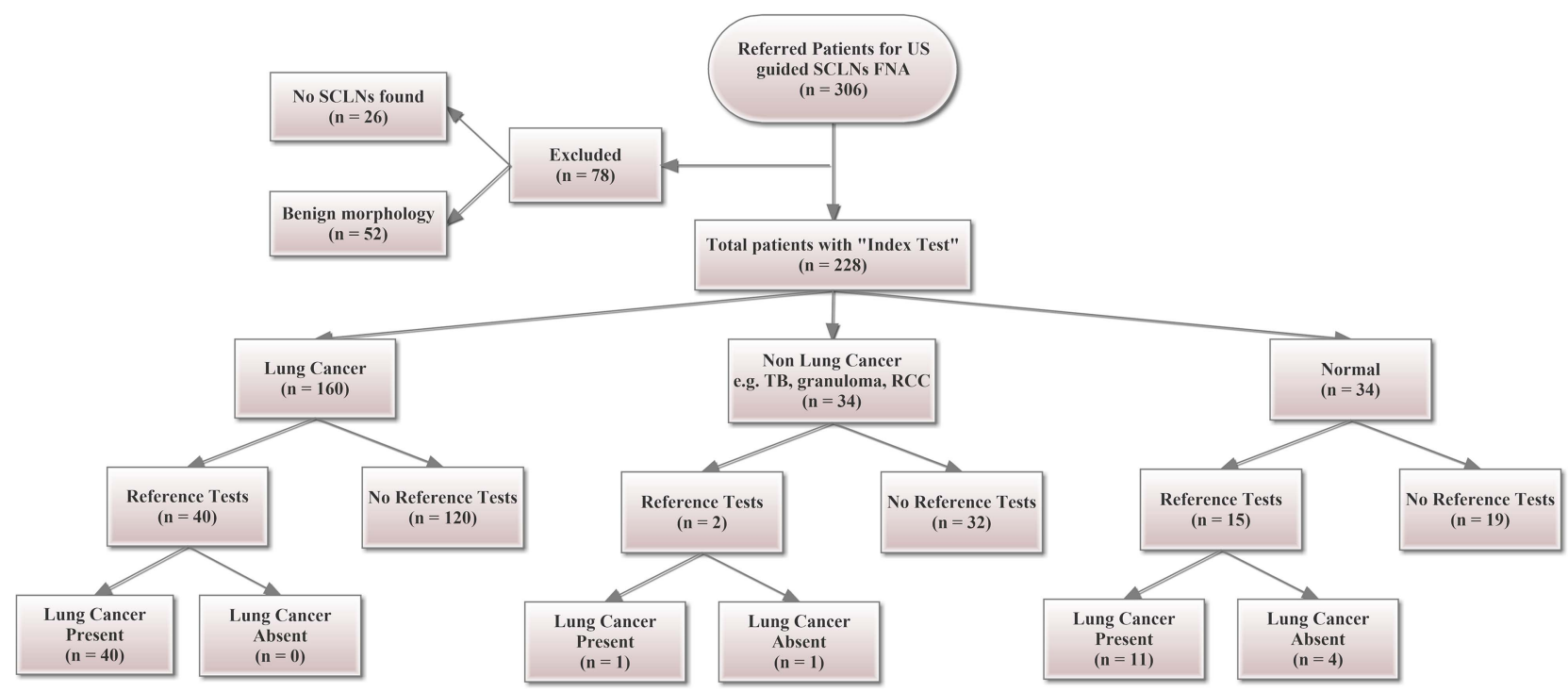

Figure 4 STARD initiative flow chart showing the distribution of US-guided SCLN FNA (Index Tests) overall diagnoses based on the total cohort of eligible patients and their subsets of abnormal results necessitating further 'Reference Tests'. FNA, fine-needle aspiration; TB, tuberculosis; RCC, renal cell carcinoma; SCLN, supraclavicular and cervical lymphadenopathy; US, ultrasound. 
NSCLC with metastatic SCL nodes detected by clinical assessment, CT or PET-CT.

The cost of US of the neck as an outpatient procedure is reported to be $£ 53$ per unit ( $£ 39-60$ upper and lower quartiles)..$^{5}$ This is approximately $32 \%$ of the average cost of TBNA and $1.7 \%$ the average cost of a mediastinoscopy inclusive of elective inpatient admission. Provided that the sensitivity seen in our study can be achieved, it is thus very likely that this procedure would be more cost-effective than TBNA and other more expensive procedures. It should also be noted that where samples might be insufficient, it is relatively easy to repeat US of the neck FNA compared with the other tests. Several studies have investigated the feasibility of EGFR analysis in cell block-based specimens from EBUS or Endoscopic Ultrasound (EUS) FNAs. A major recent study has compared cytological samples and tissue samples (approximately more than 47000 samples) from three different laboratories/studies. The study has shown that more than one-third of these samples were cytological and frequently the only available samples suitable for EGFR mutation testing for many patients. ${ }^{7}$

In a systematic review of 30 studies, da Cunha Santos et $a l^{18}$ have shown that EGFR mutation analysis can be reliably tested in NSCLC using several methods on cytological samples in addition to real-time PCR (eg, DNA sequencing or fluorescence in situ hybridisation). Another larger systematic review of 33 studies conducted by Ellison et at has recently confirmed the suitability of cytological samples for testing EGFR status. A similar opinion has been proposed in another prospective review by Shim $e t a l,{ }^{19}$ which represents the proposed guidelines from the Korean society of pathologists. Shim et al study recommends US of the neck cytology to be an established test for its rapid accrual of cancer cases in evaluating chemotherapy cycles or trial involvement.

With the increasing use of second-line and third-line systemic therapy, and the recognition that tumour biology may change during the course of the illness, there is an increasing need for further biopsy of tumours. This includes testing for acquired resistance to tyrosine kinase inhibitors. ${ }^{20} 21$ US of the neck offers a safe and effective method and is less distressing to patients. ${ }^{22}$ Such observations and proposals are incentives to further dedicated prospective studies and trials required for test validation. ${ }^{23} 24$

In this study, the authors acknowledge that the prevalence of LNs in bulky mediastinal lymphadenopathy was not fully investigated. This would require all patients with such findings to be tested with US neck. The study findings only report on the selected cases by the MDT rather than on all patients. We did not use Rapid On-site Evaluation (ROSE) to confirm the adequacy of samples, which is a potential limitation. As proven by previous studies, this could have improved overall sensitivity and potentially suggested whether samples were cellular enough for mutation testing. ${ }^{23}{ }^{24}$ A further limitation is that we were unable to review sonographic images in a small proportion of cases $(13 \%, \mathrm{n}=30)$ due to unsaved or inaccessible images of the aspirated nodes.

The start of routine EGFR testing on all adenocarcinomas and non-small cell non-squamous lung cancer cytology samples did not take place until early 2013 at Nottingham University Hospitals' histopathology laboratories. The selection of cases prior to that was made by the oncologists on an individual basis. Therefore, a total of 23 FNAC samples obtained during the study period were not EGFR tested. Also, further analysis of the follow-up data on this subgroup showed that $n=15$ have died, and $n=2$ did not want any treatment. Neither of them had any further investigations to allow for EGFR testing. Also, the latest trend in histological techniques of molecular oncogenic testing (anaplastic lymphoma kinase-ALK or KRAS) has just been introduced gradually to lung cancer workup at our institution since early 2014. Thus, none of the SCLN FNAC samples underwent any of these new tests either.

With regard to the decision of further EGFR analysis, the histopathological practice at our institution recommends further molecular analysis only when the percentage of tumour cells is $>10 \%$ of the total cells present. Automatically, $<10 \%$ will not be considered for any further molecular testing due to its invalid or poor tumour DNA (this has been observed in 5/34 EGFR testing results). Only confirmed adenocarcinomas were sent for EGFR testing, rather than all non-squamous non-small cell carcinomas (current practice). This explains why all samples were not submitted for EGFR testing. Hence, it is not possible to comment on any variability in adequacy/rates of tumour burden between subgroups. No former knowledge of the exact tumour burden triggered the latter addition of the molecular testing requests; it was suggested following the weekly MDT discussions for the management of lung cancer.

The observed EGFR testing results $(\mathrm{n}=25$ negative and $\mathrm{n}=4$ positive) showed that these samples did contain adequate tumour DNA to allow their further analysis (indicative of $>10 \%$ tumour DNA yield). Interestingly, none of the FNAC (adenocarcinoma) samples subjected to EGFR testing showed an inadequate tumour burden precluding its molecular analysis.

In summary, we have shown that the diagnostic performance of US of the neck FNA, in our hands, is good enough to recommend this test as the favoured first option in suspected lung cancer with bulky mediastinal nodes. This would offer patients a less invasive test first, and providers of healthcare a more cost-effective approach. We have also shown that the samples obtained are adequate for EGFR testing in over $80 \%$ of samples tested. For all patients to benefit, it is important to ensure that clinical services can safely and appropriately achieve high diagnostic accuracy. In addition to the many investigations used in lung cancer management, US-guided FNAC of SCLN has a potential role as a relevant staging test from oncological and radiological perspectives. It 
provides basic reaffirmatory results for staging with interesting benefits (non-radiation imaging) and by being a parallel adjunct tool to other invasive investigations, particularly in the highest stages of the disease.

\section{Author affiliations}

${ }^{1}$ Radiology Department, Nottingham University Hospitals NHS Trust, Queen's Medical Centre, Nottingham, Nottinghamshire, UK

${ }^{2}$ Histopathology Department, Nottingham University Hospitals NHS Trust Queen's Medical Centre, Nottingham, Nottinghamshire, UK

${ }^{3}$ Respiratory Medicine Unit, David Evans Research Centre, Nottingham University Hospitals NHS Trust, Nottingham, Nottinghamshire, UK

Acknowledgements Special thanks to Dr Mehluli Ndlovu, Medical Statistician at Nottingham University Hospitals, East Midlands Research Design Services (EM-RDS) for reviewing the statistical analysis in this study.

Competing interests None declared.

Provenance and peer review Not commissioned; externally peer reviewed.

Data sharing statement No additional data are available.

Open Access This is an Open Access article distributed in accordance with the Creative Commons Attribution Non Commercial (CC BY-NC 4.0) license, which permits others to distribute, remix, adapt, build upon this work noncommercially, and license their derivative works on different terms, provided the original work is properly cited and the use is non-commercial. See: http:// creativecommons.org/licenses/by-nc/4.0/

\section{REFERENCES}

1. Tiwari S, Awwad A, Kumaran M. Two year results of supraclavicular lymph node FNA for lung cancer. Lung Cancer 2013;79(Suppl 1):S21.

2. Sihoe AD, Lee TW, Ahuja AT, et al. Should cervical ultrasonography be a routine staging investigation for lung cancer patients with impalpable cervical lymph nodes? Eur J Cardiothorac Surg 2004;25:486-91.

3. Sung YM, Lee KS, Kim BT, et al. Nonpalpable supraclavicular lymph nodes in lung cancer patients: preoperative characterization with 18F-FDG PET/CT. AJR Am J Roentgenol 2008;190:246-52.

4. Hoosein MM, Barnes D, Khan AN, et al. The importance of ultrasound in staging and gaining a pathological diagnosis in patients with lung cancer-a two year single centre experience. Thorax 2011;66:414-17.

5. National Institute for Health and Care Excellence. Lung cancer: the diagnosis and treatment of lung cancer [CG121]. London: National Institute for Health and Care Excellence, National Collaborating Centre for Cancer, 2011.

6. Ellison G, Zhu G, Moulis A, et al. EGFR mutation testing in lung cancer: a review of available methods and their use for analysis of tumour tissue and cytology samples. J Clin Pathol 2013;66:79-89.
7. Hagiwara K, Kobayashi K. Importance of the cytological samples for the epidermal growth factor receptor gene mutation test for non-small cell lung cancer. Cancer Sci 2013;104:291-7.

8. Maemondo M, Inoue A, Kobayashi K, et al. Gefitinib or chemotherapy for non-small-cell lung cancer with mutated EGFR. N Engl J Med 2010;362:2380-8.

9. Mitsudomi T, Morita S, Yatabe Y, et al. Gefitinib versus cisplatin plus docetaxel in patients with non-small-cell lung cancer harbouring mutations of the epidermal growth factor receptor (WJTOG3405): an open label, randomised phase 3 trial. Lancet Oncol 2010;11:121-8.

10. Rosell R, Carcereny E, Gervais R, et al. Erlotinib versus standard chemotherapy as first-line treatment for European patients with advanced EGFR mutation-positive non-small-cell lung cancer (EURTAC): a multicentre, open-label, randomised phase 3 trial. Lancet Oncol 2012;13:239-46.

11. Bossuyt PM, Reitsma JB, Bruns DE, et al. Towards complete and accurate reporting of studies of diagnostic accuracy: the STARD initiative. BMJ 2003;326:41-4.

12. Tanaka $T$, Matsuoka $M$, Sutani $A$, et al. Frequency of and variables associated with the EGFR mutation and its subtypes. Int $J$ Cancer 2010;126:651-5.

13. Goto K, Satouchi M, Ishii G, et al. An evaluation study of EGFR mutation tests utilized for non-small-cell lung cancer in the diagnostic setting. Ann Oncol 2012;23:2914-19.

14. Gately K, O'Flaherty J, Cappuzzo F, et al. The role of the molecular footprint of EGFR in tailoring treatment decisions in NSCLC. $J$ Clin Pathol 2012;65:1-7.

15. SIGN. Management of lung cancer. Edinburgh: Health Improvement Scotland, 2014.

16. van Overhagen $\mathrm{H}$, Brakel $\mathrm{K}$, Heijenbrok MW, et al. Metastases in supraclavicular lymph nodes in lung cancer: assessment with palpation, US, and CT. Radiology 2004;232:75-80.

17. Kumaran M, Benamore RE, Vaidhyanath $\mathrm{R}$, et al. Ultrasound guided cytological aspiration of supraclavicular lymph nodes in patients with suspected lung cancer. Thorax 2005;60:229-33.

18. da Cunha Santos G, Saieg MA, Geddie W, et al. EGFR gene status in cytological samples of nonsmall cell lung carcinoma: controversies and opportunities. Cancer Cytopathol 2011;119:80-91.

19. Shim HS, Chung JH, Kim L, et al. Guideline recommendations for EGFR mutation testing in lung cancer: proposal of the Korean cardiopulmonary pathology study group. Korean $\mathrm{J}$ Pathol 2013;47:100-6.

20. Janne PA. Challenges of detecting EGFR T790M in gefitinib/ erlotinib-resistant tumours. Lung Cancer 2008;60(Suppl 2):S3-9.

21. Jackman D, Pao W, Riely GJ, et al. Clinical definition of acquired resistance to epidermal growth factor receptor tyrosine kinase inhibitors in non-small-cell lung cancer. J Clin Oncol 2010;28:357-60.

22. Soria JC, Mok TS, Cappuzzo F, et al. EGFR-mutated oncogene-addicted non-small cell lung cancer: current trends and future prospects. Cancer Treat Rev 2012;38:416-30.

23. Bozzetti C, Naldi N, Nizzoli R, et al. Reliability of EGFR and KRAS mutation analysis on fine-needle aspiration washing in non-small cell lung cancer. Lung Cancer 2013;80:35-8.

24. Bozzetti C, Negri FV, Azzoni C, et al. Epidermal growth factor receptor and Kras gene expression: reliability of mutational analysis on cytological samples. Diagn Cytopathol 2013;41:595-8. 